\title{
The Inductive Route Towards Necessity
}

\begin{abstract}
It is generally assumed that relations of necessity cannot be known by induction on experience. In this paper, I propose a notion of situated possibilities, weaker than nomic possibilities, that is compatible with an inductivist epistemology for modalities. I show that assuming this notion, not only can relations of necessity be known by induction on our experience, but such relations cannot be any more underdetermined by experience than universal regularities. This means that any one believing in a universal regularity is as well warranted to believe in the corresponding relation of necessity.
\end{abstract}

We seem to know a great deal about what is possible or not in this world. For example, I know that glass is fragile, and that if I had let a glass vase fall from my window, it would have broken. I also know, from my physics classes, that it would have accelerated at about $9.8 \mathrm{~m} / \mathrm{s}^{2}$ : in such normal conditions, an object in free fall couldn't accelerate at a much lower or higher rate. All this, it seems, is common knowledge that came to be known by experience (mine or others), not by merely reflecting on the meaning of words... Thus modal knowledge is very mundane. But it poses an epistemological challenge: how could we know, from our experience, anything about unrealized possibilities?

In this paper, I am interested in a more specific question: can relations of necessity (what is true of all possibilities) be known by mere induction?

Induction is an ampliative mode of inference by which one infers, from a sample of exemplars of a given type displaying a regularity, that this regularity holds for a larger set: the set of all objects of this type. The received view is that induction on actual experiences is insufficient to justify relations of necessity. It can only justify universal generalisation. As a consequence, empiricist-minded philosophers tend to be sceptical about natural modalities, which they consider too metaphysical, while optimists about modalities tend to accept in their epistemology ampliative modes of inference that go beyond induction, typically, inference to the best explanation.

In this paper, I wish to challenge this received view by proposing a notion of situated possibility that, if we accept it, allows for justifying relations of necessity by induction. I also wish to show that for all practical purposes, such relations of necessity are no more, no less justified than a corresponding universal generalisation, so that (accepting my framework) it would be irrational to accept the latter while rejecting the former.

The consequence of this is that empiricist-minded philosophers who are sceptical about modes of inference that go beyond induction have at their disposal a framework within which 
they can make room for modalities, while modal realists can defend their views in an empiricistfriendly way. This, I think, makes both position less uncomfortable.

My arguments assume from the start that there are possibilities of the kind considered in nature, and an empiricist might still be reluctant to accept this. Although I will give few reasons to accept them in passing, the purpose of the paper is not to argue for the existence of situated possibilities. However, it defuses one of the main arguments to reject them: their alleged principled unknowability.

Note that there has been a resurgence of empiricist epistemology for modalities in recent years. Some authors have argued in favour of the idea that knowledge of metaphysical possibilities is attainable by induction on experience (Roca-Royes, 2017: Hawke, 2011, ch. 4.2.3; Strohminger, 2015). I am sympathetic to these accounts, and mine shares similarities. However it differs in its focus on natural modalities rather than metaphysical ones, which makes it more modest in this respect. It is also more ambitious in other respects, because I aim to show that one can have knowledge not only of possibilities, but of relations of necessity from experience, and that they are as well-justified as universal regularities.

1 Induction and Underdetermination

2 Situated Possibilities

3 Why Should We Accept Situated Possibilities?

4 The Inductive Route Towards Necessity

5 Modal underdetermination

6 Modal Conflicts in Scientific Practice

7 Law-like and Accidental Generalisations

8 Conclusion

References

\section{Induction and Underdetermination}

I will assume that induction is a valid mode of inference, in the following weak sense: given that a sample of experienced situations of a given type gives us some regularity, we have prima facie good reasons to believe that this regularity can be extended to the set of all situations of the same type, so long as no relevant conflicting statement that we have knowledge of is equally justified. Hence it is often reasonable to believe statements of universal regularity. (I cast general statements in terms of experienced situations for the sake of generality, assuming that a statement like "all swans are white" can be translated into "all situations where there is one swan are situations where there is one white swan"-the notion of situation will be developed in the next section.) I do not claim that such beliefs cannot be defeated by more evidence, only that it is reasonable to believe them as long as no evidence to the contrary is at our disposal, and as long as they are not in competition with other equally well-justified statements.

The clause about competing statements is important because in principle, we could have conflicting statements that are both justified by induction. For example, an induction on gold spheres could tell us that they are stable whatever their size, and an induction on metal spheres in general could tell us that they become unstable past a given size. Since gold is a metal, these two results are in conflict. They are contrastively underdetermined by evidence. I would say that we should suspend our judgment in such a case and wait for more evidence. But I would say that there is no such problem in the case of statements that have no known serious competitors. 
It might be hard to tell what counts as a serious competitor. One might worry that there's always a possibility of coming up with an inductively justified statement in conflict with any statement, even if it is far-fetched. This problem is somehow related to Goodman (1954)'s new riddle of induction: why wouldn't the predicate blite, meaning "white before $t$ and black after t" be valid for inductive reasoning? Then "all swans are white" would be as justified as the incompatible "all swans are blite".

There are other sceptical arguments against induction, tracing back to Hume at least, that induction rests on the postulate that nature is uniform, which cannot be justified by deduction (because we need an ampliative inference) nor by induction (because it would be circular).

All the worries about induction can actually be reframed as problems of contrastive underdetermination. Hume's scepticism, for example, could be framed, taking the case of swans, as resulting from an underdetermination between the statements "all swans are white" and "all swans are white until now and non-white from now": both are compatible with the same evidence, so we cannot have any justification for one or the other without adding presuppositions.

This issue has been turned by some authors into a reductio against the kind of sceptic epistemology that would warrant induction but refrain from accepting more ambitious modes of inference, such as inference to the best explanation: if all ampliative modes of inference suffer from a problem of underdetermination, why set the bar at one place rather than another? Why not be more ambitious? This has been mounted as a defence of modal knowledge, assuming that such knowledge is arrived at by inference to the best explanation (Ladyman and Ross, 2007, ch. 2.3).

Addressing these debates is not the purpose of this paper (see Williams, 1963; Stove, 1986; Campbell, 2001; Wright, 2018, for defences of induction). I will simply assume that some statements are justified by induction while others, including "all swans are blite", are not, and I will talk of underdetermination only when two justified statements are in conflict. Like Ladyman and Ross, my focus is on modalities, but my defence of modal knowledge will take a different route. I do not wish to argue that modal sceptics place the bar at a wrong position. I wish to argue that at least for one kind of alethic modality (the one that I will present in the next section), wherever one places the bar in these matters, universal generalisations and relations of necessity will fall on the same side of it: given a universal generalisation of the type "all A are B" and its modal counterpart "necessarily, all A are B", either both are underdetermined by evidence, or neither are.

This seems counter-intuitive, because modal statements are strictly stronger than their nonmodal counterparts. My main argument is that although one statement is indeed stronger, that cannot make any pragmatic difference concerning what it is rational to believe or not, so that for all pragmatic purposes, they will always fall on the same side of underdetermination (or to be precise, one cannot be in a position to know that one of the statements is underdetermined but not the other). I think this follows at least if one assumes, as a minimal metaphysical requirement, that there are situated possibilities in the world, of a kind to be detailed in the next section. 


\section{Situated Possibilities}

Imagine a scientist wants to test the laws of classical optics. She aims a laser towards a reflecting surface from a particular angle and observes that the light ray is reflected by the surface at the same angle. I will call this a situation.

A situation is a local, bounded, occurrent state of affairs that one can experience. It can be delimited arbitrarily in space and time, as well as in terms of relevant properties and levels of precision, depending on our interests, but once this is done, it has objective characteristics (such as relative positions of objects) that are accessible to us, human beings. We describe a situation using our language, possibly using theoretical language, for example values for measured properties in a scientific context. This allows us to classify situations into types, corresponding, for example, to the kinds of objects they contain and to the way they are related to the rest of the universe, and to assign a state to them, corresponding to definite properties and relations for the objects they contain. I assume that the vocabulary we use to characterise situations is projectible, in the sense of Goodman.

It is conceivable that any given situation had been different. By this, I mean that the situation, to which we refer rigidly, could have been in a different state, that it could have had different accessible properties, while still being the same situation of the same type (with the same objects). The modality involved so far is conceptual (nearly logical), and not alethic: this is something we can imagine without contradiction. I assume that we know a priori the range of conceivable properties any given situation of a certain type could have. In the example above, given the way our situation is characterised, this would be the finite set of angles with which the laser could have aimed towards the surface, given the finite precision of our apparatus for measuring angles, and the finite set of angles at which the ray could be reflected. To take another example, if we have a thermometer capable of measuring the temperature of a liquid from 0 to 200 degrees with a precision of 1 degree, and if we put this thermometer into water in normal conditions, then any outcome in this range is a priori conceivable (even though, as we know, some outcomes are naturally impossible because water evaporates at 100 degrees). In physics, the range of conceivable possibilities would be typically represented by partitions of phase-space, or, for systems extended in time, by families of histories corresponding to conceivable measurement outcomes for degrees of freedom of the system. In more mundane contexts, we could simply enumerate these possibilities. I will refer to each of these possibilities as a conceivable situation.

Let me emphasise that conceivable situations are not conceived in abstracto, but are, so to speak, anchored to an actual situation of reference. We are not imagining any random reflecting surface and any laser pointing towards it anywhere and at any time, but this very reflecting surface and this very laser at this place and time, albeit at different angles.

It is common to think of natural (or nomic) possibilities as a sub-set of conceivable possibilities: only part of what can be conceived is naturally possible. In our example, if the laws of classical optics apply, the ray will be reflected at the same angle as the incident angle, and all other conceivable results are naturally impossible. For probabilistic theories such as statistical mechanics or quantum mechanics, there might be more than one possibility, and I assume that this is the general case.

I will refer to this sub-set of possibilities as the set of possible situations. Just as their conceivable counterparts, possible situations are anchored to a situation of reference: one could 
formalise them as a S5 modal system centred on an actual situation, limited to the objects in this situation.

Possible situations are not possible worlds. My focus on possible situations rather than possible worlds shares some motivations with situation semantics in philosophy of language. (Barwise and Perry, 1983; Kratzer, 2008) To be precise, possible situations differ from possible worlds in two important respects:

\section{Extensional limitation:}

Conceivable and possible situations are not maximal states of affairs, they are bounded in space and time, they contain a finite set of objects, they are identified by a coarse-grained type depending on our discrimination abilities and selective interests.

\section{Intensional limitation:}

As a direct consequence of extensional limitation, the range of possibilities for a situation is also limited: it could depend on environmental constraints (the rest of the universe is considered fixed) and it also depends on our discrimination abilities and interests (an epistemic agent can be "blind”, voluntarily or not, to some counterfactual variations).

These limitations are meant to ensure the compatibility of the present approach with an empiricist epistemology: they exclude from our range of alternative possibilities the ones that differ in the posited unobservable or metaphysical characteristics of the situation. They will also play a role in the arguments to follow.

In this paper, I will assume that possible situations exist. I don't want this assumption to be metaphysically loaded: I do not claim that possible situations are concrete entities, or that they represent dispositions or relations between universals. All I assume is that there are alternative ways actual situations could be, and alternative ways they couldn't be in virtue of natural and environmental constraints. I assume that it is a matter of empirical inquiry to know which, among the conceivable situations, are naturally possible and which are not, and the purpose of this paper is to show that such knowledge is attainable by induction on realized possibilities. Such knowledge will take the form of necessary statements that characterise all the possible situations of a certain type, and these alone (this time without extensional limitation).

Given the intensional limitations mentioned above, the kind of necessity at stake is presumably weaker than nomic (hence metaphysical) necessity: there could be limitations to the types of situations to which we have access given our epistemic position and discrimination abilities, so what we have is necessity of a relative kind, in the same way technical modalities are relative to our technical abilities. In the rest of this paper, I will refer to this kind of necessity as weak necessity. My aim is not to show that we could have access to metaphysical essence, let alone to the fundamental "modal structure of the world", as structural realists would have it (French, 2014; Ladyman and Ross, 2007), but only that we have access to weak necessity.

However, in contrast with the way modalities are generally considered by empiricists, I am talking about objective, alethic possibilities: not mere heuristic or fictional devices, not known a priori, not degrees of credence or other mind-dependent entities, but possibilities in the world.

\section{Why Should We Accept Situated Possibilities?}

The purpose of this paper is not to show that situated possibilities of this kind exist in nature. It is only to show that if they exist, we can have knowledge of them. Arguably, the assumption that they exist to start with, which has more metaphysical overtones, should be 
justified independently. I think that the main reasons to accept it are semantic and pragmatic, and these reasons are empiricist-friendly. There is not enough space to develop these reasons in full detail, but let me give a brief overview.

On the semantic side, modal discourse is ubiquitous in natural languages, and in science as well, and denying meaningfulness to whole parts of our discourse looks like a dogmatic position. This is particularly true for empiricists, like van Fraassen (1980), who claim to be semantic realists: most arguments in favour of semantic realism or against verificationist theories of meaning involve modalities, such as the problematic reduction of dispositional terms, or Kripke's modal argument. As far as I can see, a principled impossibility for modal knowledge is the main reason to assume that modal statements have no truth-value, but the aim of this article is to show that this reason is not tenable in the case of situated modalities. Moreover, situated modalities are well suited to account for modal discourse, since in general, counterfactual statements are not maximal and are, so to speak, "anchored" to particular objects to which we refer directly (as in Kripke (1980)'s examples-see also Kratzer (2008)).

Pragmatically, accepting modalities can help make sense of scientific practice as a rational activity. Scientists often implement experimental situations that would not occur if not with their interventions, or they vary parameters as if they were exploring various possibilities. This makes sense if their motivation is to know what would happen in such situations, implying that gaining modal knowledge must be part of the aim of science (Ladyman and Ross, 2007, p. 110). Of course, once the situations are implemented, they become actual and not merely possible. But the point is to account for their motivation prior to the implementation, or what they take the situation that they will implement to be representative of. What are they after when they create a configuration that would not occur if not with their intervention? I think the best way to make sense of their motivation is to assume that they take the situations they implement to be representative of mere possibilities. Similarly, the fact that all implemented situations are eventually actually observed does not mean that scientific knowledge is restricted to what is actually observed: implemented situations are taken to be representative of all unobserved situations of the same type.

Situated possibilities are also particularly well-suited to account for this pragmatic aspect in a minimal way, because the situations scientists implement are not maximal states of affairs.

This is not the place to develop these aspects further, and I will ask the reader to accept, for the sake of the argument, that there are situated possibilities of the kind described here in the world, that is, that there is a fact of the matter about whether the situations we encounter could have been different in one way or another, all else being equal, in virtue of external constraints on what we could experience.

\section{The Inductive Route Towards (Weak) Necessity}

Here for the preliminaries. Let us now turn to the main question of this article: can at least some relations of necessity be known by induction?

The received view, as far as I know, is that they cannot, whatever the kind of alethic necessity considered. Here is a short rationale that bolsters this view. Induction is an ampliative mode of inference by which one infers, from a sample of exemplars of a given type displaying a regularity, that this regularity holds for a larger set: the set of all objects of this type. Hence what can be known by induction is a universal regularity. But a relation of necessity goes beyond that. It does not only concern the actual world, but all possible worlds. However, we have no 
epistemic access to other possible worlds (no "modal telescope"); we do not experience relations of necessity or mere possibilities; possible worlds, if they exist as such, are causally disconnected from the actual world. An induction on possible worlds will not do: with only one exemplar at our disposal, the induction cannot get started.

If one accepts this rationale, there are two options.

The first option is to be a modal sceptic, and assume, for example, that modal talk is a mere pragmatic way of speaking that does not purport to be true or false (van Fraassen, 1989), or perhaps that modal talk is meaningful, but generally false. Taking the case of nomic necessity, one can deny that there are such things as laws of nature (which is van Fraassen's view), or one can assume that laws of nature supervene on non-modal facts, as in the best system approach (Lewis, 1973, pp. 73-77). Then laws of nature might be knowable by induction, but they have no "modal force": they merely summarise the regularities of the actual world. I have explained in the previous section why I think this modal-sceptic option is unsatisfactory.

The second option is to accept that there are other modes of inference than induction, typically, inference to the best explanation. As far as I know, this is the option followed by all philosophers who assume that laws of nature have a "modal force", and do not supervene on non-modal facts: they assume that these laws play an explanatory role, and that they are justified as such. The rationale can be the following: one sees some regularity in a sample of phenomena: all Fs in the sample are Gs; the best explanation of this observed regularity is that as a matter of nomic necessity (i.e. as a consequence of the laws of nature), all possible Fs are Gs; from this one can infer that all actual, past, present and future Fs are Gs, but one did not arrive at this result by induction: it was arrived at by inference to the best explanation. (Dretske, 1977; Armstrong, 1983; Foster, 1982)

The problem with this second option is its use of inference to the best explanation as a principle of justification. Empiricists typically deny that the non-empirical criteria that are constitutive of a good explanation, such as simplicity, are truth-conducive (this idea could be threatened by a Dutch Book argument (van Fraassen, 1989, ch. 6)). Inference to the best explanation is certainly used in science and in everyday life. It could be a heuristic device to formulate new hypotheses (which is actually how Peirce, who introduced the concept under the term "abduction", viewed it (Nyrup, 2015)), or a strategic device to decide which hypotheses to consider as a priority. But from an empiricist perspective, this tells us nothing about their validity: these hypotheses should then be put to the test so as to ensure that they are empirically adequate, and the justification of their empirical adequacy can, at best, be inductive.

Hence our two options are problematic. However I think we are facing a false dilemma: we do not have to choose between the truth-conduciveness of inference to the best explanation and modal scepticism; the received view is wrong: one can have knowledge of necessity by induction alone, at least when it comes to the weak necessity considered here.

This is because the possibilities we are considering, contrarily to nomic or metaphysical possibilities, are situated: one should think of them not in terms of possible worlds, but in terms of possible situations. Then all that is required to justify a claim of necessity by induction is to assume that the situations we experience are a representative sample of the larger set of possible situations of the same type. As an example, one take the observation that all objects released at the surface of earth accelerate towards the ground at a certain rate and infer that this is true of all possible situations of the same type, i.e. that it is true as a matter of necessity, at least relative to some background conditions (intensional limitations) that happen to be present in our 
surrounding environment. This means that one is in a position to assign truth-value to counterfactual statements such as "if I released this object, it would fall towards the earth".

An induction on possible worlds would of course be problematic, since our sample would consist in only one exemplar. If possible situations are construed as alternatives to actual ones, an induction on the alternatives to a single situation would be problematic as well, for the very same reason. But this is not so with an induction on all possible alternatives to all situations, at all places and times in the universe, assuming that observed situations are representative not only of all other unobserved actual situations of the same type, but also of all unobserved alternatives to all other situations of the same type in the universe. In this sense, the actual fall of a released object can be considered representative of the counterfactual fall of an object that was not actually released (assuming this object could have been released, which can also be known by induction on objects of this type). (Note that this account is not very different, in spirit, from Roca-Royes (2017)'s similarity-based epistemology for de re modalities.) This kind of induction is defeasible. New evidence might prove a relation of necessity wrong. But the same goes for any kind of induction.

This approach can be qualified, in Hale (2003)'s terminology, as a possibility-first approach: the idea is that knowledge of a given class of possibilities, and in particular, the absence of some relevant possibilities in this class, informs us about relations of necessity. Here, the relevant class of possibilities is the class of realized possibilities (note that Hale explicitly rejects this option, but (i) he does not discuss inductive reasoning in his paper, and (ii) he is mainly concerned with knowledge of metaphysical modalities, and assumes, wrongly I think, that knowledge of weaker modalities must derive from it).

The assumption that observed situations are representative of possible situations is not itself justified by induction. But this is no more a problem than it is for other versions of induction, where one assumes that observed situations are representative of all unobserved ones of the same type in the universe.

As we can see, assuming situated possibilities, there is a homogeneity between an induction towards universal regularities and an induction towards necessity: both involve generalising from a sample of situations to a larger set of the same type. One could reject the latter while accepting the former assuming some disparities between the two cases: either merely possible situations do not exist while unobserved actual situations do, or observed situations are representative of unobserved actual ones, but not of merely possible ones. But assuming that possible situations do exist (qua possible states of affairs), claiming that observed situations are representative only of actual ones seems to be question-begging. Actual situations are merely a sub-set of the possible situations: the ones that are realized. There seems to be no more reason to assume a bias in the way these are selected among the larger set of possible situations than there is to assume a bias in the way actually observed situations are selected among the larger set of observable ones.

The fact that, in this view, knowledge of necessity is arrived at by induction allows one to defuse a lot of arguments against the possibility of modal knowledge. Take, for example, Hume's contention that no necessary connections are found in experience. No universal regularity is found in experience either; yet universal regularities can be justified by induction, so in our case, Hume's argument against modal knowledge boils down to his argument against induction in general.

Another contention cited above is that possible worlds are causally disconnected from the actual world: we have no "modal microscope" to observe mere possibilities. However, situated 
possibilities are not disconnected from actual situations in the same way possible worlds are. Arguably, possible situations are among the possible effects of actual ones. And for this reason, we possess a sure way to explore possible types of situations: all we have to do is implement them through controlled interventions. If we want to know what would happen if a laser were aimed at a reflecting surface at an angle of precisely 32 degrees, all we have to do is to implement the situation. And it will be representative of all exemplars of its type.

One could argue that once the situation is implemented, it becomes actual rather than merely possible, and more generally, that all situations that we will ever experience are actual, so that we never have knowledge of mere possibilities. But the same problem affects an extension to all actual situations: all situations we will ever experience are observed, so we have no knowledge of unobserved ones. Yet we are generally willing to extend our knowledge of observed phenomena to unobserved, but observable phenomena. This is nothing but ampliative reasoning. So again, this type of argument affects any version of induction.

Finally, relations of necessity have been considered illegitimate by some empiricists because they are posited as explanations of empirical regularities, and inference to the best explanation, as we have seen, is generally rejected by empiricists. This is not the case here: relations of weak necessity are not explanations, but mere extensions of regularities to the realm of possibilities. They are not arrived at by inference to the best explanation, but by induction. Relations of weak necessity supervene on the mosaic of possible facts in the same way universal generalisations supervene on the mosaic of actual facts.

In sum, assuming that there are situated possibilities in the world, (weakly) necessary statements are in the same boat as universal generalisations with regards to epistemic justification. There is no principled obstacle for modal knowledge from the perspective of someone who accepts the validity of induction.

\section{Modal Underdetermination}

So far, I have argued that knowledge of weak necessity can be arrived at by induction. However, one might remain a modal sceptic on the grounds that these relations of necessity are strictly stronger than universal generalisation, by adopting a principle of metaphysical parsimony. In this section, I wish to argue that it is actually irrational to refrain from accepting induction towards necessity once induction towards universal generalisation is accepted, because no practical underdetermination could affect any relation of necessity without thereby affecting the corresponding universal generalisation. This means that the former is as warranted as the latter, from the same evidence and with the same inferential standards, at least once one accepts that there are situated possibilities.

My focus here is on contrastive underdetermination. Let us say that two statements are underdetermined if they are incompatible (they cannot be both true), but no observation can help us decide which one is true (the range of relevant observations, for example, only past observations, or all past, present and future observations, or all possible observations of actual phenomena, will determine different kinds of underdetermination: one can set the bar at various positions; my argument does not depend on a fixed choice in these matters, the point being that any choice will result in necessary statements and universal generalisations falling on the same side of it). In the following, I will only consider underdetermination of known hypotheses, where all competing hypotheses must be confirmed by induction. This amounts to assuming that it is rational to believe that a hypothesis that has no known competitors is true (we should not 
worry about unconceived alternatives, or even conceived ones when nothing confirms them). This is quite liberal, but I think this liberal attitude is acceptable in a context where one assumes, as suggested in a previous section, that our beliefs are defeasible.

I consider that the statements that can be justified by induction are perfectly general: they do not refer to specific objects, and they are not restricted to particular places or times (in logical parlance, they contain no proper names). They concern all situations of a certain type in the universe. As an example, the law of optics that states that rays of light aiming at reflecting surfaces from an angle are reflected at the same angle is general in this sense, because it does not mention any specific reflecting surface, ray of light, place or time in the world: it supposedly applies to any such configuration, anywhere and at any time.

Consider such a general statement of regularity $r_{0}$ and its modal counterpart $m_{0}$. As an example, $r_{0}$ could be the statement that all actual gold spheres of more than one kilometre in diameter in the universe are stable, and $m_{0}$ the statement that all possible gold spheres of more than one kilometre in diameter are stable. Arguably, $m_{0}$ is strictly stronger than $r_{0}$ because it quantifies over all possible situations. It would seem, then, that $m_{0}$ could be underdetermined by evidence when $r_{0}$ is not. I wish to show that this kind of case is practically impossible (or more precisely that it is impossible to know that we are in such a case), so that it can never be rational to believe a universal generalisation while rejecting the corresponding relation of necessity. Let us examine what it would look like.

Concretely, this means that $m_{0}$ could have a competitor that is as well-justified inductively, call it $m_{0}^{\prime}$, so that it is impossible to decide which of $m_{0}$ and $m^{\prime}{ }_{0}$ is true on empirical grounds. $m$ '0 could be, for example, the statement that all possible metal spheres of more than one kilometre in diameter are unstable, and it would be justified by observations of metal spheres (excluding gold spheres), while the incompatible $m_{0}$ would be justified by observations of gold spheres.

But we also want the non-modal statements not to be underdetermined. If $r_{0}$ is not underdetermined, then $r_{0}^{\prime}$, the non-modal counterpart of $m_{0}^{\prime}$, must not be a legitimate competitor of $r_{0}$. This can be the case either if $r_{0}^{\prime}$ is not as well-justified as $r_{0}$, or if it is not actually incompatible with $r_{0}$. But there can be no reason that $m_{0}^{\prime}$ is justified by induction while the weaker $r_{0}^{\prime}$ is not, so this must be because $r_{0}$ and $r_{0}^{\prime}$ are not actually in conflict: both can be true at the same time. How is this possible? Well, in our example, this is possible if there are no gold spheres of more than one kilometre in diameter in the whole actual universe. Then it is true that all actual gold spheres are stable $\left(r_{0}\right)$, and it is also true that all are unstable $\left(r_{0}^{\prime}\right)$, because there are none. The universal generalisations are not in conflict, but the corresponding modal statements are, because we are not in a position to know whether such a gold sphere would be stable or unstable if it existed. So it seems perfectly possible that two modal statements be underdetermined while their non-modal counterparts are not.

So far, modal scepticism seems vindicated: maybe metaphysical parsimony could enjoin us to reject induction towards necessity, in particular if it were always possible to find competitors to modal statements that are not in competition with their non-modal counterparts (which remains to be shown). But let us examine this case more closely: how could we possibly know that no big gold spheres exist in the whole universe? What if an alien civilisation in a remote galaxy is creating big gold spheres? What if human scientists in the distant future attempt to 
create big gold spheres, just to know whether they are stable? What if they form naturally in some places in the universe? Are there any reasons to exclude these possibilities?

Call $r_{1}$ the statement that no gold spheres exist in the universe, past present and future. Two cases should be distinguished. Either we know $r_{1}$ by induction, or we know it by direct observation. The latter case is implausible: $r_{1}$ is itself a universal generalisation (all situations are such that there are no gold spheres), and there is no way we could observe all parcels in the universe to make sure that no gold spheres exist. So we must have acquired this knowledge by induction. But now consider $m_{1}$, the modal equivalent of $r_{1}$, that is, the claim that big gold spheres are impossible. If induction justifies $r_{1}$, why couldn't it justify $m_{1}$ ? And if it does, then we have no underdetermination of the modal statements after all, because $m_{0}$ and $m_{0}^{\prime}$ are actually compatible: all possible big gold spheres are stable, and all are also unstable, because big gold spheres cannot possibly exist in our universe.

What could compel us to assume that $r_{1}$ is justified, but not $m_{1}$, is if there could be an underdetermination affecting $m_{1}$ but not $r_{1}$. But we can apply the same argument recursively, and an infinite regress looms: we must assume that a statement $m_{2}$ is underdetermined while a statement $r_{2}$ is not. Where will it stop?

There are good reasons to think that it will stop at a point where neither $m_{i}$ nor $r_{i}$ can be known to be true. In our example, I would argue that we reach this point at the first step: we should not assume that there are no big gold spheres in the universe. But even if it doesn't stop there, it couldn't go very far.

Recall that induction requires extending a regularity in a sample of situations of a given type to the set of all situations of the same type. The relevant type for $r_{0} / s_{0}$ is, in our example, situations with gold spheres with a diameter of more than one kilometre. $r_{1} / s_{1}$ claim that there is no situation of this type. Therefore the relevant type for $r_{1} / s_{1}$ must be a less specific type, a "super-type": it could be situations corresponding to gold spheres of any size (the claim being that they are never bigger than one kilometre in diameter). If we lose specificity at each step in the recursion, at some point, we will reach a completely unspecified type of situation (perhaps something like: any chunk of matter). But the less specific the type of situation is, the more various its exemplars are, and the less representative our sample can be. At some point, induction cannot be vindicated at all (this can be shown in a Bayesian framework, assuming a principle of indifference to fix prior probabilities for the conceivable states of a given type of situation). Given the size of the universe, it's very likely that many configurations of chunks of matter that we never observe in our surroundings actually form somewhere, so not observing them does not mean anything about whether they exist or not in the universe. This is a reason to assume that there could be big gold spheres in the universe, even if we never observe them.

So the regression must stop at a point where neither $m_{i}$ nor $r_{i}$ are known to be true, which means that both $m_{i-1}$ and $r_{i-1}$ are underdetermined, and neither is known to be true, which means, by recursion, that both $m_{0}$ and $r_{0}$ are underdetermined. In other words, if a modal statement is underdetermined, then its non-modal counterpart must be underdetermined as well. The reason is the following: if there can be a possible situation where two necessary statements are in conflict, then for all we know, this possible situation might as well be realized, hence the two corresponding universal generalisations are in conflict as well.

My argument rests on an example for the sake of presentation. However, it can be generalised to any universal generalisation, in so far as we consider perfectly general statements 
(not restricted to particular places and times in the universe). The argument can be formalised as follows:

1. Hypothesis: we are justified in believing that at most one of two general statements $s$ and $s^{\prime}$ is true in all possible situations. However, we don’t know which is false (they are underdetermined).

2. Hypothesis: we are justified in believing, by induction, that $s$ and $s^{\prime}$ are true in all actual situations ( $s$ is not a competitor of $s^{\prime}$ for actual situations).

3. If at most one of $s$ and $s^{\prime}$ can be true in all possible situations, then they make incompatible claims for at least one type of situation, and this type has possible instances.

4. If $s$ and $s^{\prime}$ are true in all actual situations, then any type of situation for which they make incompatible claims has no actual instance.

5. By $\underline{1}, \underline{2}, \underline{3}$ and $\underline{4}$, we are justified in believing (by induction and inference) that some type of situation for which $s$ and $s^{\prime}$ make incompatible claims has possible instances, but no actual instance.

6. We cannot be justified in believing, by induction and inference, that some type of situation has possible instances, but no actual instance.

7. Contradiction $(\underline{1}, \underline{2}, \underline{5}, \underline{6})$ : the conjunction of hypotheses $\underline{1}$ and $\underline{2}$ is absurd.

The argument rests on a few assumptions. Assumption $\underline{3}$ amounts to saying that the only justification that at most one of two statements is true in all possible situations, when we don't know which is true or false, is that they make incompatible claims for at least one possible situation. Indeed, such knowledge cannot be a posteriori (because we do not know which of the statements are true or false), so it must be a priori, which means that these statements must be a priori incompatible for some type of situation. However an a posteriori component remains (that this type of situation must be possible), and this is what drives the rest of the argument. Assumption $\underline{4}$ should be uncontroversial: incompatible claims cannot both be true. Step $\underline{5}$ rests on the principle of transmission of epistemic justification by inference, and although there are counterexamples to this principle (Wright, 1986), arguably, the present case is unproblematic.

The crucial assumption is $\underline{6}$. Its justification is roughly the following: the claim that some type of situation $T$ has no actual instance can be translated into the claim that a statement $s_{1}$ is true of all actual situations of a less specific type. But $s_{1}$ is justified by induction only if it has no known competitors, and by recursively applying the same argument, we can show that this entails that the claim that $T$ has no possible instance is as well-justified, by the same standard.

A direct consequence of the conclusion is the following: if we should believe in a universal regularity because it has no known competitor (hyp. $\underline{2}$ is true), then the corresponding relation of necessity has no known competitor (hyp. 1 is false), and we should also believe it, by the same standard.

\section{Modal Conflicts in Scientific Practice}

The argument outlined in the preceding section is rather abstract and formal, and one could perhaps wonder if it is really relevant to real cases. I think it is.

It is important to note that the argument has a normative flavour. It is an argument about what it is rational to believe. The point is not about whether necessary statements can be absolutely underdetermined by all actual facts in the universe. The answer is: of course, this can happen (for example if, actually, there are no big gold spheres in the universe). The point is 
about whether it can be rational, at some point in any enquiry, to assume that a weak necessary statement is underdetermined while the corresponding universal generalisation is not. And the answer is: no.

Despite the rather formal nature of the argument, I think it is relevant for practical enquiry, and for scientific practice in particular.

For the purpose of illustration, imagine that two groups of scientists each believe in one of our hypotheses about gold and metal spheres $s$ and $s^{\prime}$ respectively: one group, specialised in gold, has performed many tests on gold and has come to believe that all gold spheres are stable, while the other, specialised in metal, has tested many different metals and believes that since gold is a metal, some of the gold spheres (those that are big enough) are unstable. Would it be rational, in these circumstances, to assume that $s$ and $s^{\prime}$ are both true because they are both justified by different groups of scientists? Would it be rational to assume, as a direct consequence of the conjunction of $s$ and $s^{\prime}$, that there are no big gold spheres in the whole universe, even if no one ever attempted to justify that claim independently? This seems absurd. Would it be rational to remain agnostic on whether possible big gold spheres are stable or not, because we have no "modal microscopes" anyway? Should we stop our enquiry there? It seems not.

My intuition is that we could have been justified in believing either $s$ or $s^{\prime}$ in isolation. But for the first group of scientists, the fact that $s^{\prime}$ is justified by another group constitutes new evidence that $s$ might not be true after all: it acts as a potential defeater of $s$; and the inverse is true for the second group. The fact that they are modal competitors is enough to put them in conflict. What is rational, in consequence, is to pursue our enquiry: someone should make the relevant tests to know which of $s$ or $s^{\prime}$ is true. We should try to create big gold spheres.

What if we discover, during the process, that gold spheres of one kilometre in diameter cannot be created? Then the underdetermination is resolved: big gold spheres are both stable and unstable because they cannot exist. And this resolves the modal underdetermination as well: big gold spheres are impossible in this universe.

What this example purports to show is that in practice, in science or elsewhere, modal incompatibility is enough to create a conflict to be resolved. So making sure that a general hypothesis is true involves eliminating all its known modal competitors, which, incidentally, resolves any modal underdetermination. And as a consequence, we have all reasons to accept that all inductively justified knowledge has a modal force.

Now there might be some cases where we have good inductive reasons to believe that particular types of situations are never instantiated in the whole universe. Arguably, this would be a case where a mere possibility is underdetermined, while the corresponding universal generality can be known. Imagine, for example, that there is not enough gold in the whole universe to ever form gold spheres of more than one kilometre in diameter.

Is it enough to consider such cases to be a modal sceptic?

A first problem with this strategy is that it could imply modal facts. In our example, we could know that there is not enough gold in the universe because we know something about the initial conditions of the universe, and we accept a principle of conservation of matter. But the idea that the initial conditions of the universe, plus some law, constrain the kind of situations there are now is modally loaded. Arguably, grounding modal scepticism on modal knowledge is a bit problematic... 
A second problem is that this kind of case is rather limited. It does not warrant modal scepticism in general, but only for specific cases, where we have information about some type of situation that is never actually instantiated.

And a third, fatal problem in our context is that these cases only exclude knowledge about possibilities that couldn't even happen in our universe. This means that in the framework of situated possibilities, they should be considered impossible. Remember that we are concerned with alternative ways actual, bounded situations could be, given some fixed background conditions (and arguably, the initial conditions of the universe are part of these background conditions). The alternatives to a situation are situations of the same type: they contain the same objects, but these are configured differently. By this definition, if there is not enough gold in the universe, then gold spheres of more than one kilometre in diameter are impossible, and whether such gold spheres would be stable or not should not bother us: the notion of possibility involved here is too metaphysical to ever be tested.

But for all the more mundane hypotheses, that is, the ones that concern objects to which we do have epistemic access, there is no real problem. If a general hypothesis has no known competitor, then it is legitimate to extend it to all possibilities. And if it turns out that it has a modal competitor, then we should doubt this hypothesis and make relevant tests. In all cases, the relation of necessity is as well-justified as the universal generalisation.

\section{$7 \quad$ Law-like and Accidental Generalisations}

Let me finish this article with a quick note about the epistemology of laws of nature.

There is an ongoing debate about laws of nature between Humeans, who assume that laws supervene on the mosaic of actual facts, and non-Humeans, who assume that they have a "modal force", to which we could add anti-realists, who assume that there are no such things.

One could say that the arguments put forward in this paper are in line with the views of Humeans and anti-realists when it comes to scepticism about inference to the best explanation. Law-like generalisations are merely the kind of statements that can be justified by induction; no other mode of inference is needed. But my arguments fit with non-Humeanism when it comes to the idea that law-like generalisations have a modal force. It should be clear that there is no contradiction here: law-like generalisations are exactly those that can be justified by induction, and this means that they have modal force, because the corresponding law of (weak) necessity is as well-justified.

But one could worry that the present account cannot really distinguish law-like statements from accidental generalisations, if both are equally well-justified by induction. Accounting for this distinction is, after all, one of the main motives of the debate on laws of nature.

Note, however, that the kind of relation of necessity considered in this paper does not fall into the category of genuine laws of nature because it is relative to our epistemic position, and to background conditions concerning, for example, the initial state of the universe (and the exact conditions to which these relations of necessity are relative are presumably unknown). This means that the kind of necessity at stake is more permissive than nomic necessity: some regularities might count as necessary in the framework of possible situations, even though they would count as contingent from the perspective of a metaphysics of laws of nature. If, for some contingent reasons, our observations were limited to the surface of earth, the idea that all objects in free fall accelerate towards the centre of the earth at $9.8 \mathrm{~m} / \mathrm{s}^{2}$ would count as weakly 
necessary (and arguably, it could have been considered as such some time ago!), although, as we well know, it is not a universal law of nature.

This relative permissiveness can defuse the problem of demarcating accidental generalisations from law-like generalisations. My take on this issue is similar to Goodman's: I would say that identifying law-like statements is only a matter of deciding which kinds of statements are justified by induction and which are not. I assume that our capacity to imagine legitimate modal competitors for the various hypotheses we could form, and our ability to test them by implementing the relevant experimental situations, is enough to eliminate accidental generalisations. So I might invent a contrived predicate by which I show, inductively, that all coins in my pocket right now are 10 cent coins (with sufficient imagination, I might eliminate any reference to particular places and times), and it looks as if I have inductively justified an accidental generalisation to be a matter of necessity. But I can as easily justify the competing statement that all coins in pockets in general are possibly mixed. This statement has far more occurrences to make its case, and my first claim, with its tiny sample base, appears to be quite weak in comparison. And I can easily defeat my first claim by running an experiment to test which of the two competing hypotheses is true: simply by adding a 20 cent coin to my pocket.

The idea is that most accidental generalisations could be eliminated, simply because they would not survive the fierce competition to which a good enquiry would submit them. But in virtue of the permissiveness mentioned above, some other cases, often given in the literature as cases of accidental generalisations, could count as weak necessary connections in the present framework: for example, it might well be impossible for ducks to fly at more than $100 \mathrm{~km} \mathrm{~h}$, given some environmental constraints (although one might doubt that a statement about ducks is perfectly general if it must refer to a particular common ancestry to understand what a duck is). In sum, relaxing the metaphysical conditions for what counts as law-like generalisation, but maintaining rather stringent epistemic criteria for what is inductively justified, can help make sense of a meaningful distinction between accidental and law-like generalisation.

The question remains why this statement about ducks, if it is law-like, does not derive from or figure in any scientific theory, as opposed to the claim, of the same form, that no object travels faster than light. My answer would be the following: what induction towards weak necessity can justify is not fundamental scientific laws directly, but rather observational laws. Fundamental scientific laws are fairly idealised and rarely directly instantiated in our observations (Cartwright, 1983; Giere, 1999). Perhaps they can be justified by a meta-induction on observational laws, and then they might inherit their modal force from the observational laws, or perhaps they merely play a pragmatic, unifying role, without being strictly true. I won't commit myself on this issue. In any case, we seem to know a lot of law-like modal facts, about the fragility of glass for example, without necessarily grounding this knowledge in fundamental scientific theories, and this is what matters here: that these facts, with all their modal force, are perfectly knowable from experience.

\section{Conclusion}

If one accepts that there are situated possibilities in the world, that is, alternative ways actual situations could be given some natural and environmental constraints, then there is an inductive route towards knowledge of relations of necessity. Realized possibilities can be taken to be representative of merely possible ones, and they inform us about what is possible or not in this universe. The modality involved is weaker than metaphysical or nomic necessity, but that 
should not bother us for all practical purposes. Furthermore, there is no reason to resist knowledge of weak necessity, because any underdetermination that would affect it, from our perspective, would affect the corresponding universal generalisation just as much: if there is a problematic possibility that contradicts a relation of necessity, for all we know, it might well be realised somewhere in the universe.

Given that the alleged impossibility of knowledge of relations of necessity from experience remains one of the main reasons for modal scepticism, this means that there is no good reason for an empiricist not to embrace natural modalities.

\section{References}

Armstrong, David (1983). What is a Law of Nature?, volume 96. Cambridge University Press.

Barwise, Jon and Perry, John (1983). Situations and Attitudes, volume 78. Mit Press.

Campbell, Scott (2001). Fixing a hole in the ground of induction. Australasian Journal of Philosophy, 79(4), 553 - 563.

Cartwright, Nancy (1983). How the Laws of Physics Lie, volume 34. Oxford University Press.

Dretske, Fred I. (1977). Laws of Nature. Philosophy of Science, 44(2), 248-268.

Foster, John (1982). Induction, Explanation and Natural Necessity. Proceedings of the Aristotelian Society, 83, 87-101.

French, Steven (2014). The Structure of the World: Metaphysics and Representation. Oup Oxford.

Giere, Ronald N. (1999). Science without laws. Science and its conceptual foundations. University of Chicago Press, Chicago. London.

Goodman, Nelson (1954). Fact, Fiction \& Forecast, volume 25. [London]University of

Hale, Bob (2003). Knowledge of Possibility and of Necessity. Proceedings of the Aristotelian Society (Hardback), 103(1), 1-20.

Hawke, Peter (2011). Van Inwagen’s Modal Skepticism. Philosophical Studies, 153 (3): 351--64.

Kratzer, Angelika (2008). Situations in natural language semantics. In Angelika Kratzer, editor, Stanford Encyclopedia of Philosophy.

Kripke, Saul (1980). Naming and Necessity. Harvard University Press.

Ladyman, James and Ross, Don (2007). Every Thing Must Go: Metaphysics Naturalized. Oxford University Press.

Lewis, David (1973). Counterfactuals. Blackwell Publishers.

Nyrup, Rune (2015). How Explanatory Reasoning Justifies Pursuit: A Peircean View of IBE. Philosophy of Science, 82(5), 749-760.

Roca-Royes, Sonia (2017). Similarity and Possibility: An Epistemology of de Re Possibility for Concrete Entities. In Modal Epistemology After Rationalism, pages 221-45. Bob Fischer and Felipe Leon.

Stove, David (1986). The Rationality of Induction, volume 23. Oxford University Press.

Strohminger, Margot (2015). Perceptual Knowledge of Nonactual Possibilities. Philosophical Perspectives, 29(1), 363-75.

van Fraassen, Bas (1980). The Scientific Image. Oxford University Press. 
van Fraassen, Bas (1989). Laws and Symmetry, volume 102. Oxford University Press.

Williams, Donald Cary (1963). The Ground of Induction. New York, Russell \& Russell.

Wright, Crispin (1986). Facts and Certainty. Proceedings of the British Academy, 71, 429-472.

Wright, John (2018). An Epistemic Foundation for Scientific Realism: Defending Realism Without Inference to the Best Explanation. Springer International Publishing, Cham. 